\title{
Research on the reliability model of the water-entry process of air-launched vehicle under the simulation environment of numerical wave tank
}

\author{
Nuo Zhao ${ }^{1, ~ a ~, ~ L u ~ C h e n ~}{ }^{2, b}$ and Huaping Liu ${ }^{3, c}$ \\ ${ }^{1}$ China Shipbuilding Information Center, Beijing, China 100012 \\ ${ }^{2}$ Beihang University, School of Reliability and Systems Engineering, Beijing, China 100191 \\ ${ }^{3}$ Harbin Institute of Technology, School of Energy Science and Engineering, Harbin, China 150001 \\ agoingahead@sina.com, bchenxiaolu@buaa.edu.cn, ${ }^{\circ}$ hgdlhp@163.com
}

Keywords: the water-entry process of air-launched vehicle; reliability model; numerical wave tank; fitting analysis.

Abstract. The high cost of the reliability test for water-entry process of air-launched vehicle prompts the numerical simulation method which has become a mainstream in the process of equipment development. In this paper, the author integrated the simulation results of vehicle failure mechanism with the calculation of mission reliability of water-entry vehicle through the model research of water-entry process of air-launched vehicle under the condition of complex wave, and structured water-entry vehicle reliability model under complex wave condition, based on failure mechanism simulation environment. Furthermore, based on the basic flow field data obtained from CFD simulation, the author gained the movement parameters models on Attitude Angle, Angular Velocity and Centroid Displacement of the water-entry process of air-launched vehicle through the least squares fitting, which provides the basic input data for the reliability analysis of the vehicle water-entry process. In the end, the author fitted simulation results into the reliability model, and presented the reliability analysis result according to the fitting results of Attitude Angle, Angular Velocity and Centroid Displacement.

\section{Introduction}

Air-launched vehicle has become a mainstream of "Air-Land-Ocean" combat mode, which demands that the vehicle can launch rapidly and reliably in all kinds of complicated sea and weather conditions. Establishing numerical wave environment conform to actual situation and applying the simulation data to implement reliability analysis model is the key point of this simulation research. So far, in the air-launched vehicle simulation field, Senhu Li ${ }^{[1]}$, Bin Xia ${ }^{[2]}$, Zhen Chen ${ }^{[3]}$ and Yong Wang ${ }^{[4]}$ had carried out simulation studies about the water-entry process of air-launched vehicle through the finite element software; Xuenong Chen ${ }^{[5]}$, Jiasheng Zhou ${ }^{[6]}$, Qi Sun ${ }^{[7]}$ and Jun Wang ${ }^{[8]}$ had conducted numerical simulations about the water-entry process of air-launched vehicle, and discussed the influence of different control parameters on the vehicle water-entry process separately. However, these models lacked comprehensive consideration of the coupling mechanism of various affecting factors on system reliability analysis, which affected the reliability of the simulation results to some extent.

Based on vehicle movement parameters under different numerical wave parameters and the database of structural vibration deformation parameters, the author took into consideration various factors such as sea conditions, wave phases, direction angles, and launch speed/attitude/structural strength, and established the response model among movement parameters, structure parameters and wave parameters. Integrated probabilistic method and non-probabilistic methods into this response model, the author analyzed failure modes of vehicle water-entry ballistic reliability, attitude reliability and structure reliability, and established the reliability forecasting model for water-entry process of air-launched vehicle. 


\section{Simulation Analysis for Reliability Model}

The establishment of a high quality numerical wave tank. In order to ensure the waves can fully flow, the volume of the established numerical wave tank is $(100 \times 25 \times 35) \mathrm{m} 3$. Figure 1 shows the numerical wave tank and initial position of air-launched vehicle. Vehicle's head is vertical from the still wave surface 6 meters away (the same as length of the vehicle) before air-launched. Then the vehicle moves as linear accelerated motion along with the direction 30 degrees to the horizontal, and starts free-flying after close to the phase interface and the speed reach to $25 \mathrm{~m} / \mathrm{s}$. Considering that the air-launched vehicle may produce slamming during the water-entry process, the author conducted intensive processing ( 2.3 million) to the grids around vehicle and its moving area, as shown in Figure 2.

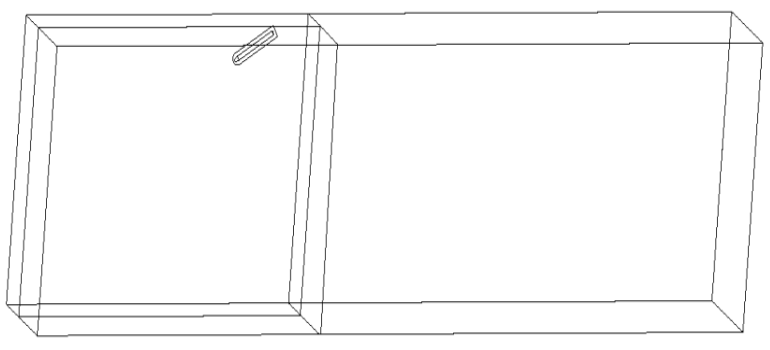

Figure 1. Vehicle's initial position in the tank.

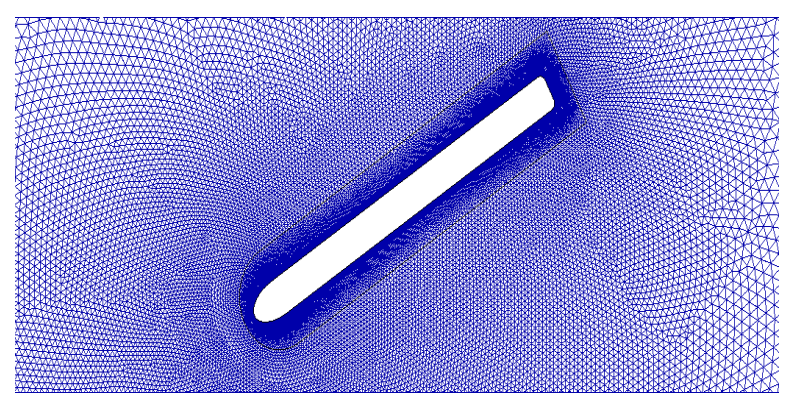

Figure 2. Grid distribution map

The simulation of reliability model under numerical wave tank. After consider the factors about sea conditions (wave characteristic parameters) and wave phases, the author added the influence analysis about water-entry angle, which means the smaller included angle between vehicle's axis and the horizontal. In order to improve the simulation efficiency and save time, different variables were controled seperately, 3 levels of sea conditions, 4 phases of wave and 2 kinds of water-entry angles were selected, which produced 10 kinds of simulation scheme of water-entry process of air-launched vehicle, as shown in Table 1.

Table 1. Simulation scheme of water-entry process of air-launched vehicle

\begin{tabular}{lclllll}
\hline Schemes & $\begin{array}{l}\text { Levels of sea } \\
\text { conditions }\end{array}$ & $\begin{array}{l}\text { Wave } \\
\text { height } \\
(\mathbf{m})\end{array}$ & $\begin{array}{l}\text { Wave } \\
\text { length } \\
(\mathbf{m})\end{array}$ & $\begin{array}{l}\text { Wave-length } \\
\text { period (s) }\end{array}$ & $\begin{array}{l}\text { Wave } \\
\text { phases }\end{array}$ & $\begin{array}{l}\text { Water } \\
\text {-entry } \\
\text { angle }\end{array}$ \\
\hline Scheme1 & 3th & 0.88 & 15 & 3.0996 & $0^{\circ}$ & $30^{\circ}$ \\
Scheme2 & 3th & 0.88 & 15 & 3.0996 & $180^{\circ}$ & $30^{\circ}$ \\
Scheme3 & 4th & 1.35 & 30 & 4.3835 & $0^{\circ}$ & $30^{\circ}$ \\
Scheme4 & 4th & 1.35 & 30 & 4.3835 & $180^{\circ}$ & $30^{\circ}$ \\
Scheme5 & 5th & 1.80 & 40 & 5.0616 & $0^{\circ}$ & $30^{\circ}$ \\
Scheme6 & 5th & 1.80 & 40 & 5.0616 & $90^{\circ}$ & $30^{\circ}$ \\
Scheme7 & 5th & 1.80 & 40 & 5.0616 & $180^{\circ}$ & $30^{\circ}$ \\
Scheme8 & 5th & 1.80 & 40 & 5.0616 & $270^{\circ}$ & $30^{\circ}$ \\
Scheme9 & 5th & 1.80 & 40 & 5.0616 & $0^{\circ}$ & $60^{\circ}$ \\
Scheme10 & 5th & 1.80 & 40 & 5.0616 & $180^{\circ}$ & $60^{\circ}$ \\
\hline
\end{tabular}

Based on the high quality numerical wave tank, the evolution mechanism of unsteady flow field characteristics and hydrodynamic characteristic for water-entry process of air-launched vehicle influenced by different sea conditions, wave phases and water-entry angles is discussed. Then, the changing regularity of motion parameters, attitude, strength and structural deformation of the vehicle under different wave environments and launch conditions was analyzed based on numerical calculation results. Specifically, the 
changing curves of wave flow field, water pressure and motion parameters (Displacement, Velocity, Angular Velocity and Attitude Angle, etc.) of water-entry process of air-launched vehicle were simulated, when 3 levels of sea conditions coupling with 4 wave phases and 2 water-entry angles. Then, the changes of different directions' forces and torques of vehicle was analyzed, and the water-entry process of air-launched vehicle reliability model under complex wave condition, based on failure mechanism simulation environment was established.

\section{The Establishment and Validation of Reliability Model for Water-entry Process of Air -launched Vehicle under Simulation Environment}

Reliability model for vehicle's water-entry process under simulation environment. To establish the water-entry process reliability model for air-launched vehicle, we need to consider both water-entry environment and initial conditions, let us define I as

$I=\left\{i^{1}=\right.$ wave-condition, $i^{2}=$ wave-phase, $i^{3}=$ centroid-initial-velocity, $i^{4}=$ water-entry-angle $\}$.

We can conclude the water-entry process reliability model for air-launched vehicle as Equations 1 .In there, vehicle motion state parameters are composed of Attitude Angle, Angular Velocity and Centroid Displacement. According to this, we can get fault criterion of water-entry process based on simulation mechanism as Equations 2.

$$
\begin{aligned}
R(t)= & F\left(t_{s}, t_{u}, Y, S, I\right) \\
= & \int^{R^{n}} p_{Y}\left(t_{u} \mid Y_{I} \in \Phi_{I}\right) p_{I}\left(t_{s}, t_{u}, I \in R^{n} \mid I\right) \\
= & p\left(t_{s}, t_{u} \mid i^{1}=0.5\right) \int_{0}^{360} \int_{0}^{1} p_{Y}\left(t_{u} \mid Y_{I} \in \Phi_{I}\right) p\left(i^{2}=x\right) p\left(i^{4}=y\right) d x d y \\
& +p\left(t_{s}, t_{u} \mid i^{1}=0.67\right) \int_{0}^{360} \int_{0}^{1} p_{Y}\left(t_{u} \mid Y_{I} \in \Phi_{I}\right) p\left(i^{2}=x\right) p\left(i^{4}=y\right) d x d y \\
& +p\left(t_{s}, t_{u} \mid i^{1}=0.83\right) \int_{0}^{360} \int_{0}^{1} p_{Y}\left(t_{u} \mid Y_{I} \in \Phi_{I}\right) p\left(i^{2}=x\right) p\left(i^{4}=y\right) d x d y
\end{aligned}
$$

$$
Y_{I} \in \Phi_{I}=\left\{\begin{array}{l}
\theta_{I}^{1} \in \Phi_{I}^{1} \\
\omega_{I}^{2} \in \Phi_{I}^{2} \\
y_{I}^{3} \in \Phi_{I}^{3}
\end{array}=\left\{\begin{array}{l}
\theta_{I}^{1} \geq r_{I}^{\theta} \\
\omega_{I}^{2} \geq r_{I}^{\omega} \\
y_{I}^{3} \geq r_{I}^{y}
\end{array}\right.\right.
$$

Based on the simulation result of water-entry process of air-launched vehicle, we can deduce the Attitude Angle fitting formula Equations 3, where $i^{1}$ is sea condition level and $i^{2}$ is wave phase, both of them are dimensionless parameters. In detail, $i^{1}=i_{0}^{1} / 6$, the $i^{1}$ value of 3 th, 4 th and 5 th level sea condition are $0.5,0.6667$ and $0.8333 ; i^{2}=i_{0}^{2} / 360$, the $i^{2}$ value for wave crest phase and wave trough phase are 0 and 0.5 .

$$
\begin{aligned}
\theta_{I}^{1} & =\theta\left(i^{1}, i^{2}\right) \\
& =\left[-0.4459-0.1427 \times\left(i^{2}-0.25\right)+0.5005 \times\left(i^{1}-0.6667\right)-2.4238 \times\left(i^{1}-0.6667\right) \times\left(i^{2}-0.25\right)\right. \\
& \left.+1.4358 \times\left(i^{1}-0.6667\right)^{2}+12.0869 \times\left(i^{1}-0.6667\right)^{2} \times\left(i^{2}-0.25\right)\right] \times T^{3} \\
& +\left[0.7547+0.4192 \times\left(i^{2}-0.25\right)-0.8406 \times\left(i^{1}-0.6667\right)+4.5387 \times\left(i^{1}-0.6667\right) \times\left(i^{2}-0.25\right)\right. \\
& \left.-3.1981 \times\left(i^{1}-0.6667\right)^{2}-22.5287 \times\left(i^{1}-0.6667\right)^{2} \times\left(i^{2}-0.25\right)\right] \times T^{2} \\
& +\left[-0.3418-0.2687 \times\left(i^{2}-0.25\right)+0.3794 \times\left(i^{1}-0.6667\right)-2.2024 \times\left(i^{1}-0.6667\right) \times\left(i^{2}-0.25\right)\right. \\
& \left.+1.8289 \times\left(i^{1}-0.6667\right)^{2}+11.2123 \times\left(i^{1}-0.6667\right)^{2} \times\left(i^{2}-0.25\right)\right] \times T \\
& +\left[0.03120+0.03159 \times\left(i^{2}-0.25\right)-0.03391 \times\left(i^{1}-0.6667\right)+0.2112 \times\left(i^{1}-0.6667\right) \times\left(i^{2}-0.25\right)\right. \\
& \left.-0.1999 \times\left(i^{1}-0.6667\right)^{2}-1.1230 \times\left(i^{1}-0.6667\right)^{2} \times\left(i^{2}-0.25\right)\right]
\end{aligned}
$$


In the same way, Equations 4 is the Angular Velocity fitting formula, and Equations 5 is the Centroid Displacement formula obtained by fitting. In the formula, the specific meaning of various parameters (dimensionless), wave phases and its definition of positive or negative are all in accordance with the above Attitude Angle fitting analysis. The positive direction of $\mathrm{X}$ axis in the same with wave propagation direction, and the positive direction of $\mathrm{Y}$ axis is vertical up.

$$
\begin{aligned}
& \omega_{I}^{2}=\omega\left(i^{1}, i^{2}\right) \\
& =\left[-1.9229+1.0151 \times\left(i^{2}-0.25\right)-0.8289 \times\left(i^{1}-0.6667\right)+2.2590 \times\left(i^{1}-0.6667\right) \times\left(i^{2}-0.25\right)\right. \\
& \left.-9.5605 \times\left(i^{1}-0.6667\right)^{2}-78.1424 \times\left(i^{1}-0.6667\right)^{2} \times\left(i^{2}-0.25\right)\right] \times T^{3} \\
& +\left[2.7506-1.2671 \times\left(i^{2}-0.25\right)+1.9807 \times\left(i^{1}-0.6667\right)-2.8162 \times\left(i^{1}-0.6667\right) \times\left(i^{2}-0.25\right)\right. \\
& \left.+15.0132 \times\left(i^{1}-0.6667\right)^{2}+159.7805 \times\left(i^{1}-0.6667\right)^{2} \times\left(i^{2}-0.25\right)\right] \times T^{2} \\
& +\left[-1.0169+0.4816 \times\left(i^{2}-0.25\right)-1.0976 \times\left(i^{1}-0.6667\right)+1.2495 \times\left(i^{1}-0.6667\right) \times\left(i^{2}-0.25\right)\right. \\
& \left.-6.9019 \times\left(i^{1}-0.6667\right)^{2}-85.2304 \times\left(i^{1}-0.6667\right)^{2} \times\left(i^{2}-0.25\right)\right] \times T \\
& +\left[0.07330-0.04320 \times\left(i^{2}-0.25\right)+0.1223 \times\left(i^{1}-0.6667\right)-0.1407 \times\left(i^{1}-0.6667\right) \times\left(i^{2}-0.25\right)\right. \\
& \left.+0.6655 \times\left(i^{1}-0.6667\right)^{2}+8.9475 \times\left(i^{1}-0.6667\right)^{2} \times\left(i^{2}-0.25\right)\right] \\
& y_{I}^{3}=y\left(i^{1}, i^{2}\right) \\
& =\left[-2.0003-3.999 \times\left(i^{2}-0.25\right)+7.5023 \times\left(i^{1}-0.6667\right)-29.9994 \times\left(i^{1}-0.6667\right) \times\left(i^{2}-0.25\right)\right. \\
& \left.+45.0225 \times\left(i^{1}-0.6667\right)^{2}+107.9676 \times\left(i^{1}-0.6667\right)^{2} \times\left(i^{2}-0.25\right)\right] \times 10^{-4} \times X^{3} \\
& +\left[-3.5003-3.1986 \times\left(i^{2}-0.25\right)+8.4021 \times\left(i^{1}-0.6667\right)-40.7957 \times\left(i^{1}-0.6667\right) \times\left(i^{2}-0.25\right)\right. \\
& \left.+37.8227 \times\left(i^{1}-0.6667\right)^{2}+251.9770 \times\left(i^{1}-0.6667\right)^{2} \times\left(i^{2}-0.25\right)\right] \times 10^{-3} \times X^{2} \\
& +\left[56.9150-0.2598 \times\left(i^{2}-0.25\right)+1.2754 \times\left(i^{1}-0.6667\right)-6.8990 \times\left(i^{1}-0.6667\right) \times\left(i^{2}-0.25\right)\right. \\
& \left.+9.4542 \times\left(i^{1}-0.6667\right)^{2}+49.3175 \times\left(i^{1}-0.6667\right)^{2} \times\left(i^{2}-0.25\right)\right] \times 10^{-2} \times X \\
& +\left[2.4901+1.7192 \times\left(i^{2}-0.25\right)-4.2915 \times\left(i^{1}-0.6667\right)+24.5974 \times\left(i^{1}-0.6667\right) \times\left(i^{2}-0.25\right)\right. \\
& \left.-32.9543 \times\left(i^{1}-0.6667\right)^{2}-150.4658 \times\left(i^{1}-0.6667\right)^{2} \times\left(i^{2}-0.25\right)\right] \times 10^{-2}
\end{aligned}
$$

Model validation. The definition of wave phases were given in Figure 3.Take the end of the vertical acceleration upward movement as time zero, at this moment, vehicle's head 2 meters vertical from the still wave surface. Observed from the positive direction of $\mathrm{Z}$ axis, the attitude angle is on positive direction when vehicle spin anticlockwise while on negative direction when spin along with clockwise, and attitude angle remain unchanged $(\theta=0)$ when the $\mathrm{Z}$ axis parallel to the vertical direction, as shown in Figure 4.

If the vehicle entries numerical wave tank under 3th level sea condition and in the wave crest phase (sea condition $i^{1}=0.5$, wave phase $i^{2}=0$ ), the head contacted with wave surface is about the time $t_{s}=0.90 \mathrm{~s}$ and the 
tail finished the water-entry is about the time $t_{u}=1.14 \mathrm{~s}$. Similarly, the corresponding parameters of other conditions are shown in Table 2.

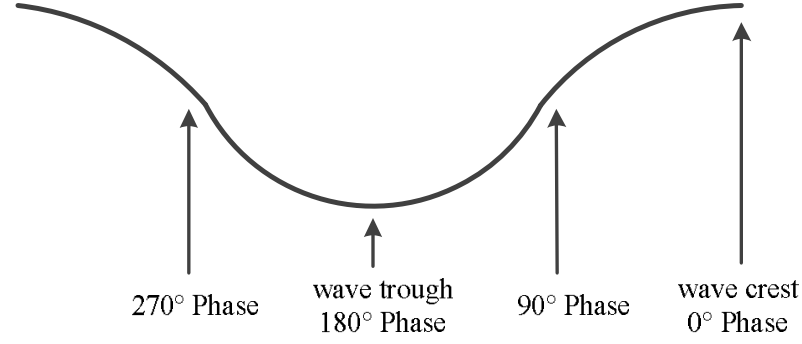

Figure 3. The definition of wave phases

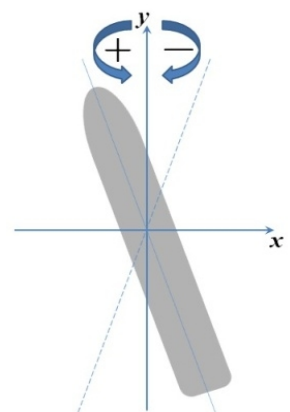

Figure 4. The direction of Attitude Angle

Table 2. Parameters of different water-entry conditions

\begin{tabular}{|l|c|c|c|c|}
\hline \multicolumn{1}{|c|}{ water-entry conditions } & $\boldsymbol{i}^{\mathbf{I}}$ & $\boldsymbol{i}^{2}$ & $\boldsymbol{t}_{\boldsymbol{s}}$ & $\boldsymbol{t}_{\boldsymbol{u}}$ \\
\hline 3th level sea condition \& wave crest phase $\left(0^{\circ}\right)$ & 0.5 & 0 & $0.90 \mathrm{~s}$ & $1.14 \mathrm{~s}$ \\
\hline 3th level sea condition \& wave trough phase $\left(180^{\circ}\right)$ & 0.5 & 0.5 & $1.02 \mathrm{~s}$ & $1.28 \mathrm{~s}$ \\
\hline 4th level sea condition \& wave crest phase $\left(0^{\circ}\right)$ & 0.6667 & 0 & $0.87 \mathrm{~s}$ & $1.11 \mathrm{~s}$ \\
\hline 4th level sea condition \& wave trough phase $\left(180^{\circ}\right)$ & 0.6667 & 0.5 & $1.05 \mathrm{~s}$ & $1.32 \mathrm{~s}$ \\
\hline 5th level sea condition \& wave crest phase $\left(0^{\circ}\right)$ & 0.8333 & 0 & $0.80 \mathrm{~s}$ & $1.05 \mathrm{~s}$ \\
\hline 5th level sea condition \& wave trough phase $\left(180^{\circ}\right)$ & 0.8333 & 0.5 & $1.09 \mathrm{~s}$ & $1.35 \mathrm{~s}$ \\
\hline
\end{tabular}

After assigned the values of $i^{1} \& i^{2}$ corresponding to each case's water-entry conditions into the above unified equations, we obtained the theoretical fitting curves of vehicle's Attitude Angle, Angular Velocity and Centroid Displacement of each condition respectively. Figure 5 7 presented the comparison result between theoretical fitting curves and practical simulation output data.

(1) Attitude Angle fitting reliability analysis
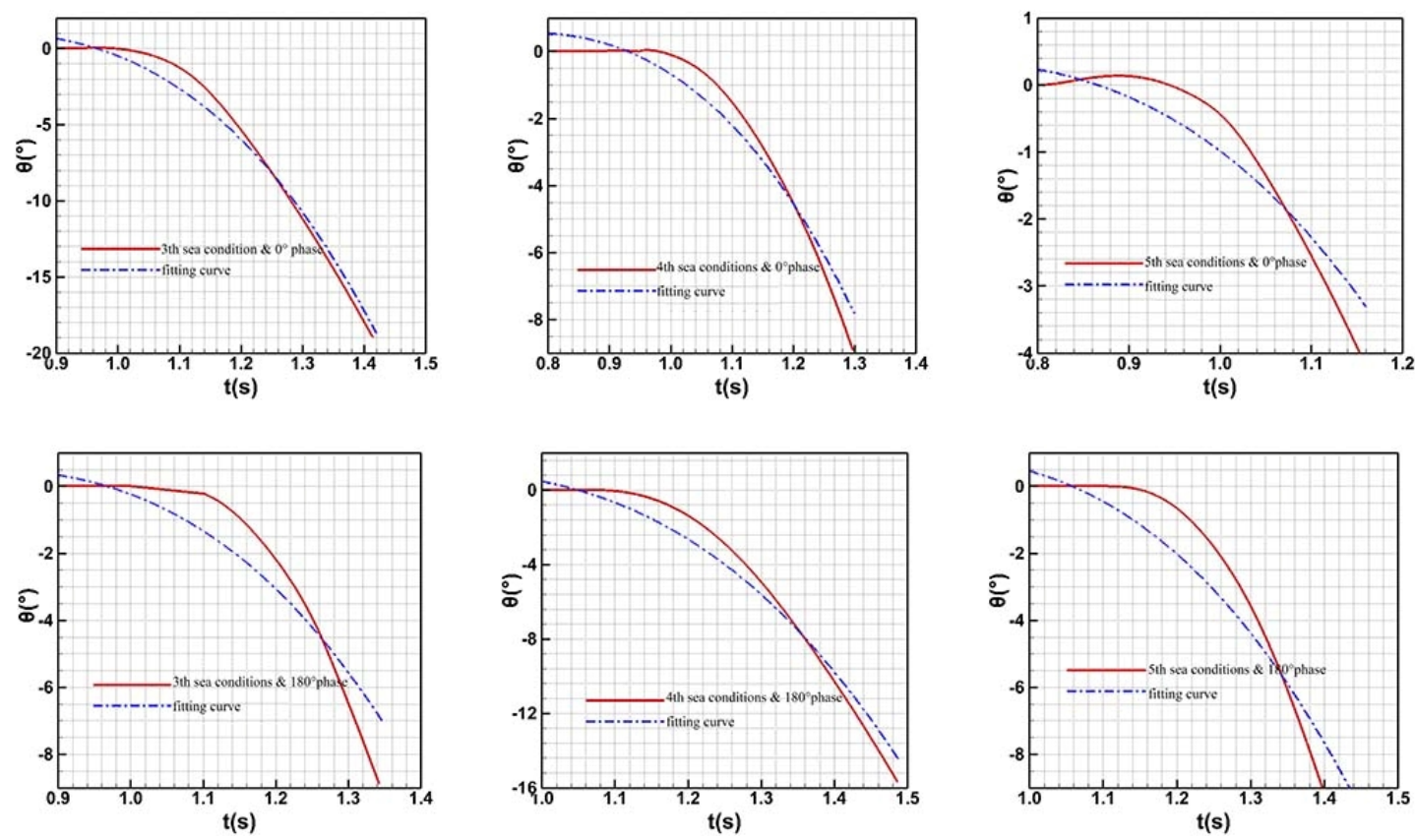

Figure 5. Comparison of Attitude Angle between fitting curve \& simulation results under different conditions. 
(2) Angular Velocity fitting reliability analysis
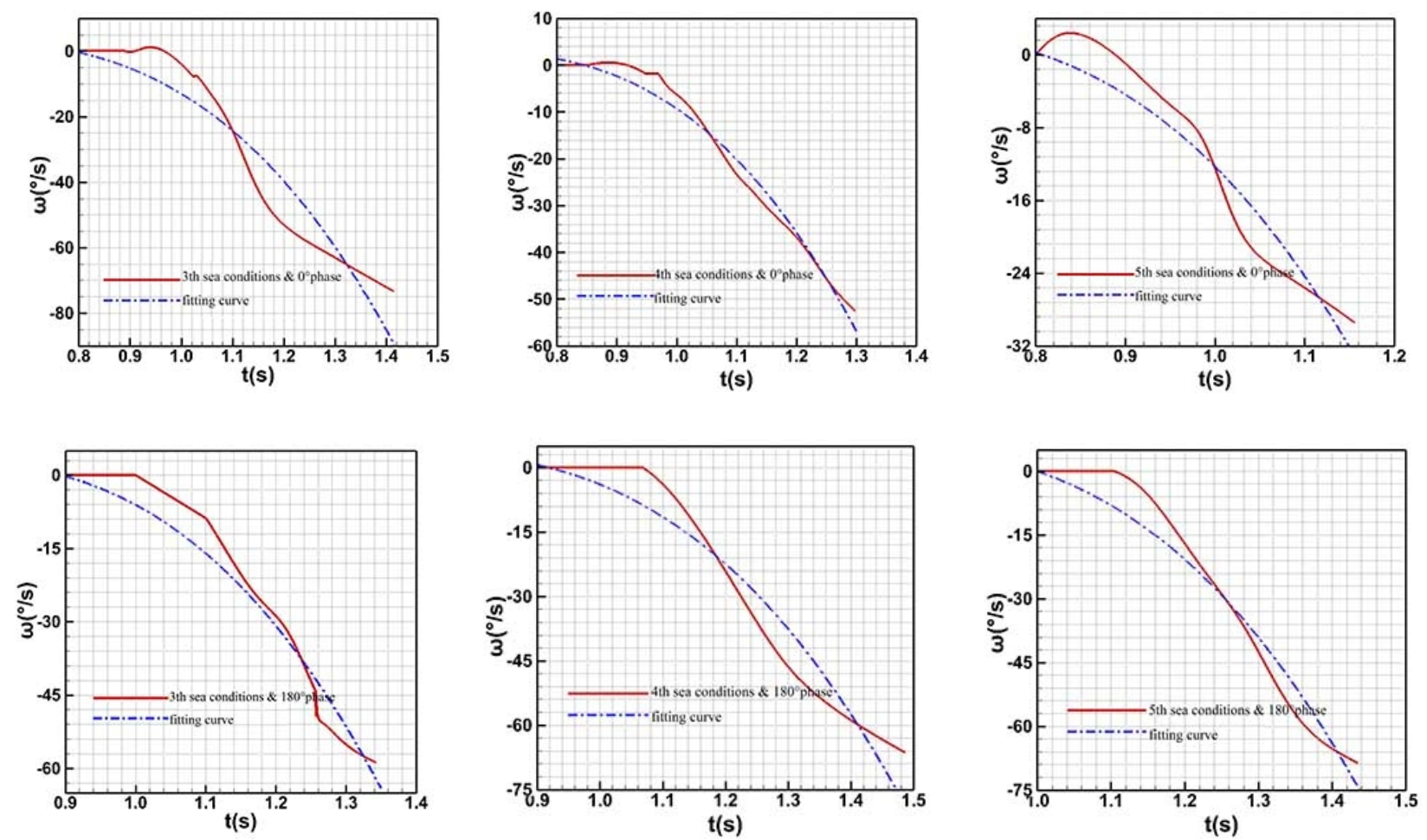

Figure 6. Comparison of Angular Velocity between fitting curve \&simulation results at different conditions.

(3) Centroid Displacement fitting reliability analysis
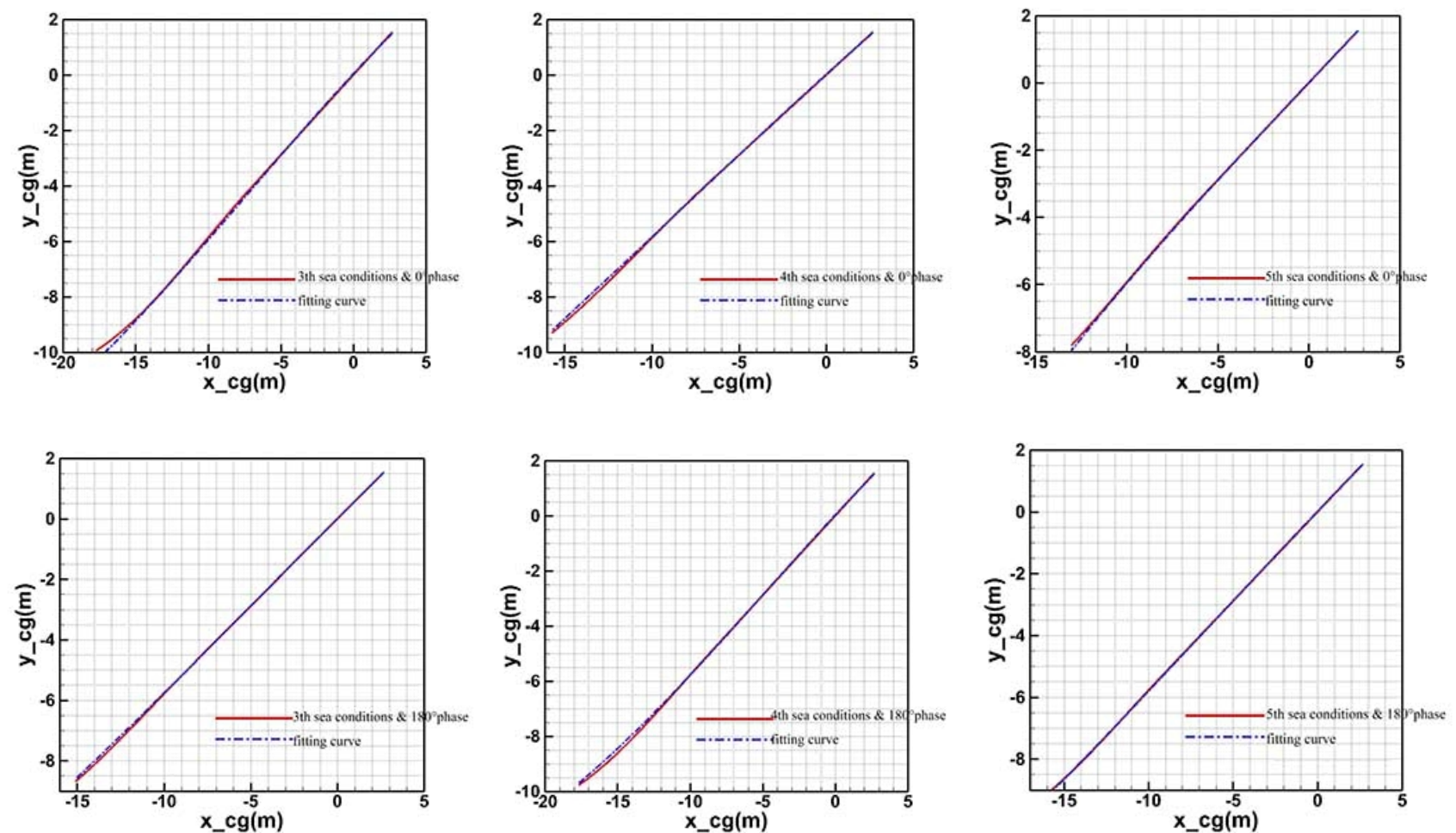

Figure7. Comparison of Centroid Displacement between fitting curves \& simulations at different conditions. 
Observed from the comparison diagrams between theoretical fitting curves and practical simulation results of vehicle's Attitude Angle, Angular Velocity and Centroid Displacement under different sea conditions \& wave phases, it's easy to find that there exists some deviations between the fitting curves and simulation results of Attitude Angle and Angular Velocity, which may cause by the slamming generated at the moment of water-entry. The simulated curves flow with more fluctuation while the overall trend of them is consistent, the deviation range is limited. However, the fitting curves and simulation results of Centroid Displacement are almost complete overlap. In general, the fitting result is acceptable, and the structured vehicle water-entry reliability model under complex wave condition based on failure mechanism simulation environment achieved a higher accuracy and completeness.

\section{Conclusion}

Based on water-entry vehicle movement parameters under different numerical wave parameters and the structural vibration deformation parameters, the response model among movement parameters, structure parameters and wave parameters is established. Integrated probabilistic method and non-probabilistic methods into this response model, the author analyzed failure mechanism of vehicle water-entry ballistic reliability, attitude reliability and structure reliability, and established the basic reliability model for water-entry process of air-launched vehicle. Combined the simulation results of vehicle failure mechanism with the calculation of mission reliability of vehicle water-entry process, the reliability model of vehicle water-entry under complex wave condition based on failure mechanism simulation environment is structured.

The movement parameters model of the water-entry process is obtained through the least squares fitting based on the basic flow field data obtained from CFD simulation, which provides the basic input data for the reliability analysis of the water-entry process of air-launched vehicle. In the end, the author fitted simulation results into the reliability model, and presented the reliability analysis result according to the fitting results of Attitude Angle, Angular Velocity and Centroid Displacement. The fitting curves and simulation results is almost overlapping which validate that the fitting result is acceptable, and the structured reliability model of the water-entry process of air-launched vehicle under the simulation environment of numerical wave tank is applicability and effectiveness.

\section{Acknowledgment}

This paper was supported by Equipment advanced research funds of GAD (General Armament Department): 9140A19020513CB44256.

\section{References}

[1] Senhu Li, Yousheng He, Journal of Hydrodynamics. 1992,7(1): 72-78. (In Chinese)

[2] Bin Xia, Zhen Chen, China Offshore Platform. 2005, 22(7): 22-28. (In Chinese)

[3] Zhen Chen, Journal of Shanghai Jiaotong University. 2007, 41(9): 1425-1428. (In Chinese)

[4] Yong Wang, Xingman Yang, Sci-tech innovation \& productivity. 2014, 10: 047. (In Chinese)

[5] Xuenong Chen, Yousheng He, Acta mechanica sinica. 1990, 22(2): 129-137. (In Chinese)

[6] Jiasheng Zhou, Wenjun Yi, Zhongyuan Wang, Journal of Projectiles, Rockets, Missiles and Guidance. 2007, 27(3): 173-178. (In Chinese)

[7] Qi Sun, Jun Zhou, Peng Lin, Journal of System Simulation. 2010, 22(6). (In Chinese)

[8] Jun Wang. The impact dynamics simulation study of torpedo water-entry [D]. Master degree theses of Kunming University of Science and Technology. 2010. (In Chinese) 